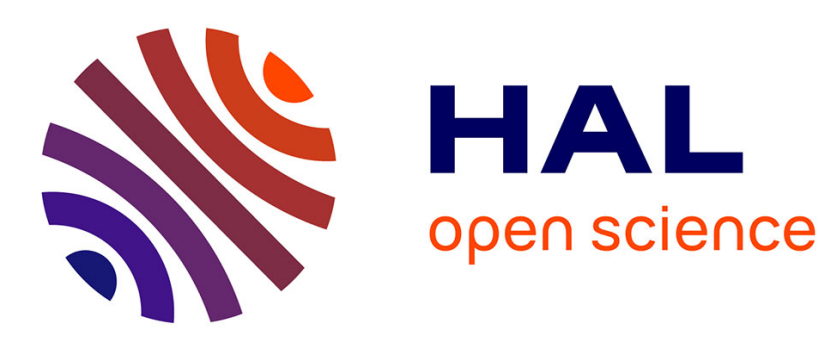

\title{
Influence of boron-interstitials clusters on hole mobility degradation in high dose boron-implanted ultrashallow junctions
}

Fabrice Severac, Fuccio Cristiano, Eléna Bedel-Pereira, Pier Francesco Fazzini, Jonathan Boucher, Wilfried Lerch, Silke Hamm

\section{To cite this version:}

Fabrice Severac, Fuccio Cristiano, Eléna Bedel-Pereira, Pier Francesco Fazzini, Jonathan Boucher, et al.. Influence of boron-interstitials clusters on hole mobility degradation in high dose boron-implanted ultrashallow junctions. Journal of Applied Physics, 2010, 107 (12), pp.123711. 10.1063/1.3446844 . hal-01922687

\section{HAL Id: hal-01922687 \\ https://hal.science/hal-01922687}

Submitted on 14 Nov 2018

HAL is a multi-disciplinary open access archive for the deposit and dissemination of scientific research documents, whether they are published or not. The documents may come from teaching and research institutions in France or abroad, or from public or private research centers.
L'archive ouverte pluridisciplinaire HAL, est destinée au dépôt et à la diffusion de documents scientifiques de niveau recherche, publiés ou non, émanant des établissements d'enseignement et de recherche français ou étrangers, des laboratoires publics ou privés. 


\title{
Influence of boron-interstitials clusters on hole mobility degradation in high dose boron-implanted ultrashallow junctions
}

\author{
Fabrice Severac, ${ }^{1, a)}$ Fuccio Cristiano, ${ }^{1}$ Elena Bedel-Pereira, ${ }^{1}$ Pier Francesco Fazzini, ${ }^{1}$ \\ Jonathan Boucher, ${ }^{1}$ Wilfried Lerch, ${ }^{2}$ and Silke $\mathrm{Hamm}^{3}$ \\ ${ }^{1}$ LAAS-CNRS, 7 avenue du colonel Roche, F-31077 Toulouse, France and UPS, INSA, INP, ISAE, LAAS, \\ Université de Toulouse, F-31077 Toulouse, France \\ ${ }^{2}$ Centrotherm thermal solutions GmbH \& Co. KG, Johannes-Schmid-Straße 8, 89143 Blaubeuren, Germany \\ ${ }^{3}$ Mattson Thermal Products GmbH, Daimlerstrasse 10, D-89160 Dornstadt, Germany
}

(Received 15 April 2010; accepted 11 May 2010; published online 21 June 2010)

\begin{abstract}
Hole mobility degradation has been studied in high-dose boron-implanted ultrashallow junctions containing high concentrations of boron-interstitial clusters (BICs), combining an empirical method based on the self-consistent interpretation of secondary-ion-mass spectrometry (SIMS) and Hall measurements and liquid-nitrogen $\left(\mathrm{LN}_{2}\right)$ to room temperature (RT) hole mobility measurements. It has been found that BICs act as independent scattering centers which have a strong impact on hole mobility in addition to the other scattering mechanisms such as lattice and impurities scattering. A mobility degradation coefficient $\alpha$ has been introduced, which gives information on the mobility degradation level in the analyzed junctions. In the case of very high concentrations of BICs (containing a boron density up to $8 \times 10^{14} \mathrm{~cm}^{-2}$ ), measured hole mobilities were found to be $\sim 40 \%$ lower than corresponding theoretical values. BICs dissolution through multiple Flash anneals at high temperature $\left(1300{ }^{\circ} \mathrm{C}\right)$ reduces the observe mobility degradation. (C) 2010 American Institute of Physics. [doi:10.1063/1.3446844]
\end{abstract}

\section{INTRODUCTION}

To continue scaling down complementary metal-oxide semiconductor devices (CMOS), ultrashallow source/drain junctions with low resistivity ${ }^{1}$ must be fabricated. As a consequence, dopants are implanted at very high concentrations that often exceed their solid solubility limit in silicon at the temperature used for their activation anneal. In the case of $p+/ n$ junctions, this results in the formation of immobile and electrically inactive clusters containing boron and silicon interstitial atoms (BICs) (Refs. 2-9) which can degrade their electrical properties. The understanding of the physical phenomena responsible for this degradation is essential for the optimization of the fabrication processes.

The assessment of the electrical dopant activation (total active dose or maximum active concentration) of a junction is often based on the combination of the chemical dopant concentration profile [measured by secondary-ion-mass spectroscopy (SIMS)] and junction sheet resistance (obtained by four point probes measurements), ${ }^{10-14}$ in which it is systematically assumed that the mobility versus concentration relations $^{15,16}$ are valid. However, nowadays junctions systematically contain a large amount of BICs that may act as additional scattering centers and have an impact on the carrier mobility. Indeed, Clarysse et al. ${ }^{17}$ observed some deviations between theoretical and measured mobilities in various samples containing BICs and suggested that inactive boron could be responsible for this difference. In addition, we showed in a recent work $^{18}$ that carrier mobility measurements based on the Hall effect are also affected by the presence of high BICs concentration. In that work, the value of

${ }^{\text {a)} E l e c t r o n i c ~ m a i l: ~ f s e v e r a c @ l a a s . f r ~}$ the Hall scattering factor $r_{H}$, the correction factor used to link raw measurements of Hall concentration and mobility to the active carrier concentration and drift mobility, was found to be in very good agreement with the existing literature ${ }^{19,20}$ in references samples without BICs $\left(r_{H}=0.74 \pm 0.1\right)$, whereas in samples containing high BICs concentration, a value of $r_{H}=0.95 \pm 0.1$ was measured. We explained this increase in terms of the additional scattering centers associated to the presence of high concentrations of BICs.

In this paper, we first show that BICs are also directly responsible of drift mobility degradation thanks to a method of analysis we established ${ }^{21}$ based on the self consistent interpretation of SIMS and Hall effect measurements of various samples. Then, we used low temperature mobility measurements to identify the mechanisms responsible for this degradation. The investigated junctions were realized using the same preamorphisation and implantation conditions but different annealings, in order to obtain various BICs quantities, and included a reference sample, realized by chemical vapor deposition (CVD) with low boron (B) concentration to avoid BICs formation.

\section{SAMPLES PREPARATION AND CHARACTERIZATION PROCESS}

For these experiments, $n$-type, $200 \mathrm{~mm}$ prime $\mathrm{Si}$ wafers of (100) orientation and $1-20 \Omega \mathrm{cm}$ resistivity were used. All wafers were implanted with $500 \mathrm{eV}^{11} \mathrm{~B}^{+}$ions to a dose of $1 \times 10^{15} \mathrm{~cm}^{-2}$ after a ${ }^{74} \mathrm{Ge}^{+}$preamorphisation step (30 $\mathrm{keV}$ to a dose of $1 \times 10^{15} \mathrm{~cm}^{-2}$ ). All implants were performed on an applied materials quantum batch implanter. Germanium was implanted under $7^{\circ}$ tilt and $22^{\circ}$ twist and boron under $0^{\circ}$ tilt and $0^{\circ}$ twist conditions. Prior to the im- 
plantation, the wafers were cleaned with Piranha, HF, $\mathrm{SC}_{1}$, and $\mathrm{SC}_{2}$ etches, and an additional $\mathrm{HF}$ dip was done less than 15 min before the implantation step.

The first sample received a low temperature annealing $\left(750^{\circ}, 15 \mathrm{~min}\right)$ under $\mathrm{N}_{2}$ flow rate. The second sample underwent a spike anneal (at $1000{ }^{\circ} \mathrm{C}$ ) in a Mattson 3000 Plus RTP system equipped with Mattson's absolute temperature measurement (a temperature controller optimized for spike anneals) and wafer rotation. The ramp-up rate to the prestabilization step was $50 \mathrm{~K} / \mathrm{s}$. The recipe included prestabilization at $650{ }^{\circ} \mathrm{C}$ for $10 \mathrm{~s}$ followed by a spike with a ramp-up rate set to $250 \mathrm{~K} / \mathrm{s}$. Three more samples were annealed with single or multiple Flash anneals (1,2 or 3$)$ in a Mattson Millios fRTPTM in a nitrogen ambient. From an intermediate temperature of $750{ }^{\circ} \mathrm{C}$ the wafer was flashed to a peak temperature of $1300{ }^{\circ} \mathrm{C} .{ }^{5}$

Finally, a reference sample was realized by CVD, on a (100) $n$-type (6-24 $\Omega \mathrm{cm}$ resistivity) $200 \mathrm{~mm}$ prime $\mathrm{Si}$ wafers at $700{ }^{\circ} \mathrm{C}$ with a pressure of 80 Torr and $\mathrm{H}_{2}$ as carrier gas in an ASM Epsilon 2000 reduced pressure single wafer CVD tool. The thickness of the deposited layer was $100 \mathrm{~nm}$ and the corresponding B concentration was $1 \times 10^{20} \mathrm{~cm}^{-3}$. As the concentration of this sample is higher than the solid solubility of boron ${ }^{22}$ at the deposition temperature, it was submitted to a spike anneal at $1050{ }^{\circ} \mathrm{C}$ to ensure a complete activation of the dopants.

SIMS analysis was performed on a CAMECA IMS 7F using a $500 \mathrm{eV} \mathrm{O}_{2}{ }^{+}$primary ions to evaluate the distribution of (B) atoms. Hall measurements were carried out on an Accent HL5500 Hall effect system and based on the Van der Pauw technique ${ }^{23}$ on Greek-cross structures realized with photolithographic processes and plasma etching.

\section{METHOD OF ANALYSIS}

For a boron doped $p+/ n$ junction, this method consists in the calculation, of the electrical parameters measured by Hall effect sheet resistance $\mathrm{R}_{\mathrm{S}}$, active dose $\left\langle N_{S}\right\rangle$, and drift mobility $\left.\left\langle\mu_{d}\right\rangle\right)$ on the basis of the dopant chemical concentration profile measured by SIMS and of the dopant mobility versus concentration relation. ${ }^{15}$ We first define from the chemical dopant profile measured by SIMS, $C(x)$, the active dopant concentration profile, $C_{a}(x)$, as follows:

$$
C_{a}(x)=\left\{\begin{array}{ll}
C_{e l} & \text { for } C(x) \geq C_{e l} \\
C(x) & \text { for } C(x)<C_{e l}
\end{array},\right.
$$

where $C_{e l}$ is the maximum active dopant concentration. In addition, we also assume that $C_{a}(x)$ is identical to the carrier concentration profile, $p(x)$ (in the case of $\mathrm{B}$ ). In fact, a difference between $C_{a}(x)$ and $p(x)$ is expected only at low concentration levels, however this difference would have a very small impact on the calculated values of $\mathrm{R}_{\mathrm{S}},\left\langle N_{S}\right\rangle$ and $\left\langle\mu_{d}\right\rangle$.

Starting from the hole concentration profile, $p(x)$, it is then possible to calculate the sheet resistance, $\mathbf{R}_{\mathrm{S}}$, of the $p$ $+/ n$ junction, if the hole drift mobility, $\mu_{p}$, versus concentration relation is known

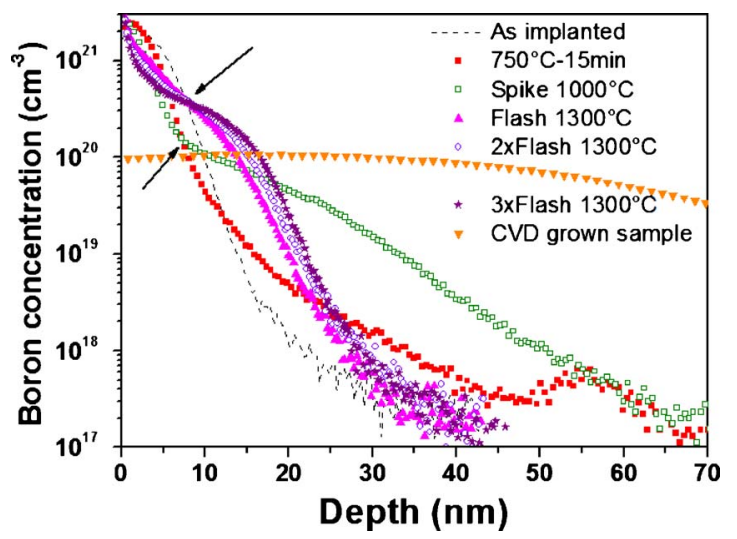

FIG. 1. (Color online) Boron SIMS profiles of the five studied samples implanted with the same conditions but submitted to various annealing; the continuous line indicate the as-implanted profile and the arrows the "kink." SIMS profile of the reference CVD grown sample is also plotted.

$$
\mathrm{R}_{\mathrm{S}}=\frac{1}{q \int_{0}^{X_{j}} p(x) \mu_{p}[p(x)] d x},
$$

where $\mathrm{q}$ is the electron charge and $X_{j}$ the junction depth.

From $p(x)$ and $\mu_{p}$, it is also possible to calculate the active dose $\left\langle N_{S}\right\rangle$ and the drift mobility $\left\langle\mu_{d}\right\rangle$, usually obtained from Hall measurements ${ }^{24}$

$$
\begin{aligned}
& \left\langle N_{S}\right\rangle=\frac{\left(\int_{0}^{X_{j}} p(x) \mu_{p}(x) d x\right)^{2}}{\int_{0}^{X_{j}} p(x) \mu_{p}^{2}(x) d x}, \\
& \left\langle\mu_{d}\right\rangle=\frac{\int_{0}^{X_{j}} p(x) \mu_{p}^{2}(x) d x}{\int_{0}^{X_{j}} p(x) \mu_{p}(x) d x} .
\end{aligned}
$$

Finally, and in order to take into account the possible impact of BICs on the carrier mobility, we make the assumption that the presence of BICs induces an additional scattering center that reduces the drift mobility.

We therefore define the effective drift mobility, $\mu_{\text {eff }}$ as

$$
\mu_{\text {eff }}=\alpha \cdot \mu_{p} \text { with } \alpha \leq 1 \text {, }
$$

where $\alpha$ is the mobility degradation coefficient. For a totally activated junction, $\alpha=1$; while in the presence of a high concentration of BICs, we expect to find, for $\alpha$, a value lower than unity. According to all previous assumptions, Eqs. (2)-(4) can therefore be rewritten substituting $p(x)$ by $C_{a}(x)$ [cf. Eq. (1)] and $\mu_{p}$ by $\mu_{\text {eff }}$ (Eq. (5)). In this way, from the SIMS profiles of the investigated samples and the theoretical values of drift mobility against carrier concentration, ${ }^{15}$ sheet resistance, active dose, and mobility can be calculated from these equations and matched to the measured values using two fitting parameters: $C_{e l}$, the maximum doping profile concentration, and $\alpha$, the mobility degradation coefficient.

\section{RESULTS AND DISCUSSION}

\section{A. Mobility coefficient $(\alpha)$ evolution as a function of BICs density}

SIMS dopant profiles of the six studied samples are reported in Fig. 1. Static peaks (i.e., immobile boron), clearly appear in the spike annealed sample at $1000{ }^{\circ} \mathrm{C}$ and in the Flash annealed samples. These peaks are delimited by 
TABLE I. Hall effect measurements and model analysis values of the $1000{ }^{\circ} \mathrm{C}$ spike annealed sample.

\begin{tabular}{lccc}
\hline \hline & $\begin{array}{c}N_{S} \\
\left(\mathrm{~cm}^{-2}\right)\end{array}$ & $\begin{array}{c}\mu_{d} \\
\left(\mathrm{~cm}^{2} / \mathrm{V} \mathrm{s}\right)\end{array}$ & $\begin{array}{c}\mathrm{R}_{\mathrm{S}} \\
(\Omega / \mathrm{sq})\end{array}$ \\
\hline Hall measurements $r_{H}=0.95$ & $2.8 \times 10^{14}$ & 35.3 & 631 \\
Model $C_{e l}=1.8 \times 10^{20} \mathrm{~cm}^{-3}, \alpha=1$ & $2.8 \times 10^{14}$ & 51.2 & 435 \\
Model $C_{e l}=1.8 \times 10^{20} \mathrm{~cm}^{-3}, \alpha=0.69$ & $2.8 \times 10^{14}$ & 35.3 & 630 \\
\hline \hline
\end{tabular}

"kinks" (showed by arrows in Fig. 1 for spike and Flash anneals in SIMS profiles), followed by diffused tails at higher depths, kinks indicate the concentration levels above which B forms BICs, close to the solid solubility of B in silicon at the corresponding annealing temperatures. The area covered by the static peaks corresponds to the total dose of clustered boron and it decreases when increasing the anneal temperature from 1000 to $1300{ }^{\circ} \mathrm{C}$ or when increasing the thermal budget at $1300{ }^{\circ} \mathrm{C}$ (from 1 to 3 pulses). The decrease in static peak area is associated with BICs dissolution. At $750{ }^{\circ} \mathrm{C}$, the low boron diffusivity makes it more difficult to distinguish between the immobile and mobile region of the boron profile. In addition, the kink concentration level $\left(\sim 4 \times 10^{19} \mathrm{~cm}^{-3}\right)$ is higher than the solid solubility at this temperature due to the enhanced activation achievable after solid phase epitaxy at low temperature. ${ }^{10}$

In all cases, the kink concentration gives an indication of the maximum active dopant concentration, $C_{e l}$ [cf. Eq. (1)]. However, for a more precise evaluation of $C_{e l}$, we combined Hall effect and SIMS measurements and calculated the measured active dose $\left\langle N_{S}\right\rangle$ [Eq. (3)] by replacing $p(x)$ with $C_{a}(x)$ [Eq. (1)] and using $C_{e l}$ as a fitting parameter as describe in previous section. For example, in the case of the $1000{ }^{\circ} \mathrm{C}$ spike annealed sample, whose measured Hall effect parameters are reported in Table I (first raw), a value of $C_{e l}=1.8$ $\times 10^{20} \mathrm{~cm}^{-3}$ is found. This value corresponds well to the "kink" position in the SIMS profile, as show in Fig. 2 and is in very good agreement with results obtained in a recent work, ${ }^{18}$ where scanning capacitance microscopy measure-

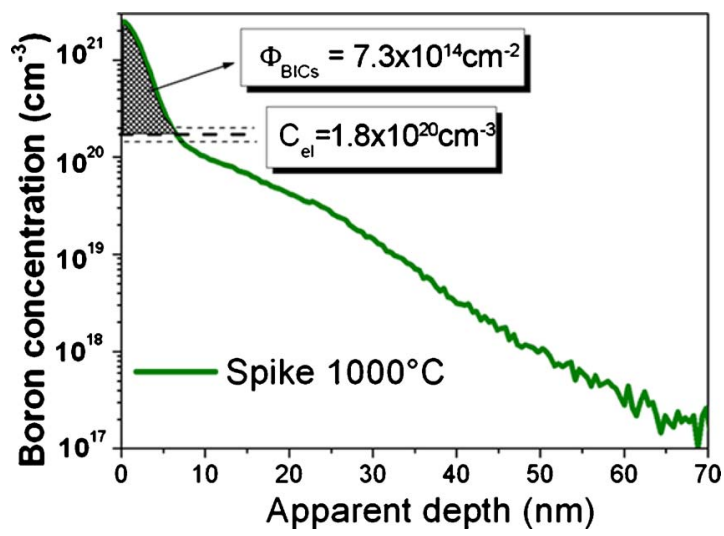

FIG. 2. (Color online) Boron SIMS profile of the $1000{ }^{\circ} \mathrm{C}$ spike annealed sample, the maximum electrical concentration $\left(C_{e l}\right)$ is found equal to 1.8 $\times 10^{20} \mathrm{~cm}^{-3}$ and the evaluated dose of BICs of this sample (hatched area) equal to $7.3 \times 10^{14} \mathrm{~cm}^{-2}$. The calculated $C_{e l}$ is close to the "kink." Measurement errors are indicated with dashed lines.
TABLE II. Various parameters determined for each sample from SIMS and Hall effect measurements, respectively, the electrical concentration $\left(C_{e l}\right)$, the measured drift mobility at room temperature, evaluated BICs doses, and mobility coefficient $\alpha$.

\begin{tabular}{lccc}
\hline \hline Sample annealing & $\begin{array}{c}C_{e l} \\
\left(\mathrm{~cm}^{-3}\right)\end{array}$ & $\begin{array}{c}\Phi_{\mathrm{BICs}} \\
\left(\mathrm{cm}^{-2}\right)\end{array}$ & $\alpha$ \\
\hline $750{ }^{\circ} \mathrm{C} 15 \mathrm{~min}$ & $1.47 \times 10^{20}$ & $8.8 \times 10^{14}$ & 0.58 \\
Spike $1000{ }^{\circ} \mathrm{C}$ & $1.80 \times 10^{20}$ & $7.3 \times 10^{14}$ & 0.69 \\
Flash $1300{ }^{\circ} \mathrm{C}$ & $5.48 \times 10^{20}$ & $4.1 \times 10^{14}$ & 0.78 \\
$2 \times$ Flash $1300{ }^{\circ} \mathrm{C}$ & $5.25 \times 10^{20}$ & $3.4 \times 10^{14}$ & 0.83 \\
$3 \times$ Flash $1300{ }^{\circ} \mathrm{C}$ & $5.31 \times 10^{20}$ & $2.5 \times 10^{14}$ & 0.86 \\
$\mathrm{CVD}$ sample & $1 \times 10^{20}$ & 0 & 1 \\
\hline \hline
\end{tabular}

ments were used to show that the kink concentration level provides a reliable indication of the maximum active concentration of the implanted boron.

Once the $C_{e l}$ is determined, we can then evaluate the total BICs density $\Phi_{\text {BICs }}$ (hatched area in Fig. 2). The same procedure was used for all the samples, and the corresponding values of $C_{e l}$ and $\Phi_{\mathrm{BICs}}$ are reported in Table II columns 2 and 3. It is important to note that we took into account, as mentioned in the introduction, the impact of BICs on Hall measurements, using the appropriate value of the Hall scattering factor (cf. Ref. 18), i.e., $r_{H}=0.95$ in samples containing BICs and $r_{H}=0.75$ in the reference sample without BICs.

We now focus on the two others parameters measured by Hall effect: sheet resistance and drift mobility. If the extracted values of $C_{e l}$ (cf. Table II) are inserted in Eqs. (2) and (4), together with the standard hole mobility versus concentration curves, ${ }^{15}$ the calculated values of the sheet resistance and drift mobility are different from the measured ones, as show in Table I (second raw) for the spike annealed sample. In particular, the calculated drift mobility for this sample $\left(51.2 \mathrm{~cm}^{2} / \mathrm{V} \mathrm{s}\right)$ is higher than the measured one. Unlike Eq. (3), used to calculate the active dose $\left\langle N_{S}\right\rangle$, Eqs. (2) and (4) (sheet resistance and drift mobility, respectively) are directly dependent on the mobility versus concentration relation, ${ }^{15}$ assuming that the presence of BICs is responsible for the degradation of carrier mobility, we therefore adjusted the value of the mobility coefficient $\alpha$ [defined in Sec. III, Eq. (5)] in order to match the measured sheet resistance and drift mobility. A single value of $\alpha(0.69)$ is found to allow a perfect matching of both measured values in the spike annealed sample (cf. Table I, 3rd row), which confirms the hypothesis of mobility degradation due to BICs, while the value of $\alpha$ gives information on this degradation level. In the same way, we calculated $\alpha$ for each studied sample and the obtained values are reported in Table II (last column). We can observe that mobility degradation linearly increases (i.e., $\alpha$ decreases) when $\Phi_{\text {BICs }}$ increases, which confirms the direct impact of BICs on drift mobility. In the case of the reference sample (CVD grown, without BICs $\left(\Phi_{\mathrm{BICs}}=0\right)$, no mobility degradation was measured and so $\alpha$ was found equal to unity. We plotted the variation in $\alpha$ as a function of $\Phi_{\mathrm{BICs}}$ in Fig. 3. Error bars were taken equal to $15 \%$ for both $\alpha$ and $\Phi_{\text {BICs }}$ due to both SIMS and Hall experimental errors.

We clearly observe that BICs dissolution occurs during annealing and is systematically linked to an increase in the 


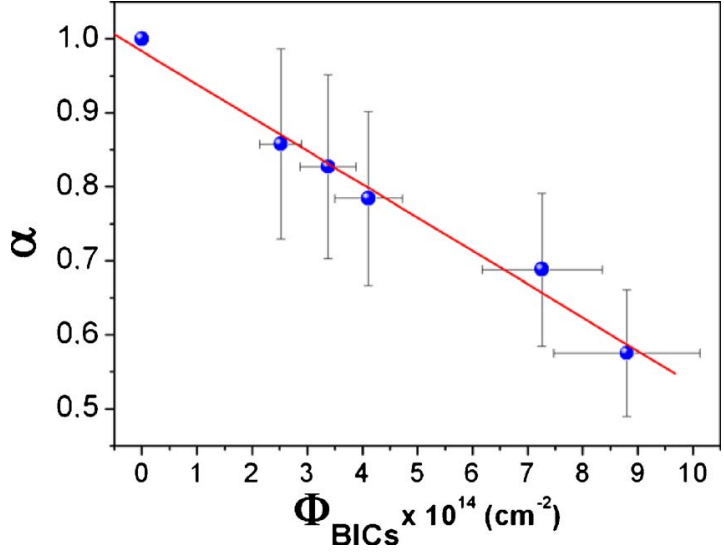

FIG. 3. (Color online) Mobility coefficient $\alpha$ function of BICs dose. The point found for the reference samples, free from any residual defects (so $\left.\Phi_{\mathrm{BICs}}=0\right)$ and without mobility degradation $(\alpha=1)$ is also reported. Improvement of mobility is clearly observed when BICs dose decrease.

mobility coefficient, which means that measured mobilities become closer to the "standard" mobilities values as measured in samples without BICs. We can therefore conclude that BICs are directly responsible for the observed mobility degradation.

In order to provide a further confirmation of this conclusion, we realized on these samples temperature-dependant drift mobility measurements (from $\mathrm{LN}_{2}$ to RT), which allow to experimentally isolate the different scattering mechanisms. ${ }^{25}$ Results are presented in Sec. IV B.

\section{B. Temperature dependence of drift mobility}

At room temperature, mobility is limited by both ionised impurities and lattice scattering; when temperature decreases, lattice vibrations are lower, leading to an initial mobility increase, while the impurity scattering mechanisms induces a mobility decrease. As a result of these two opposite trends, carrier mobility typically goes through a maximum level at a temperature which is proportional to the impurity concentration. ${ }^{26-30}$

Mobility measurements as a function of temperature (from $\mathrm{LN}_{2}$ to RT) were done by Hall effect. At RT, the measured mobility values varied from one sample to another (between 30 and $35 \mathrm{~cm}^{2} / \mathrm{V} \mathrm{s}$ ) due to the different annealing conditions, leading to different values of $C_{e l}$ and $\Phi_{\mathrm{BICs}}$ (cf. Table II). Therefore, in order to observe mobility fluctuations as a function of temperature and to interpret them more easily, we plotted in Fig. 4 normalized values with respect the one $\left(\mu_{0}\right)$ obtained at RT from the sample annealed at $750{ }^{\circ} \mathrm{C}$ for $15 \mathrm{~min}$. We can observe an increase in mobility for all the samples when the measurement temperature decreases followed by a deflection in the curves. In the case of samples containing higher densities of BICs (spike 1000 and $750{ }^{\circ} \mathrm{C}$ 15 min annealed samples), a maximum of mobility is reached at $150 \mathrm{~K}$ and $200 \mathrm{~K}$, respectively, followed by a decrease at lower temperatures. This behavior is similar to the one described above, and which is observed in samples containing different impurity concentrations. However, in our case, the investigated samples have similar dopant concentrations $\left(1.8 \times 10^{20}\right.$ and $1.5 \times 10^{20} \mathrm{~cm}^{-3}$, respectively, cf.

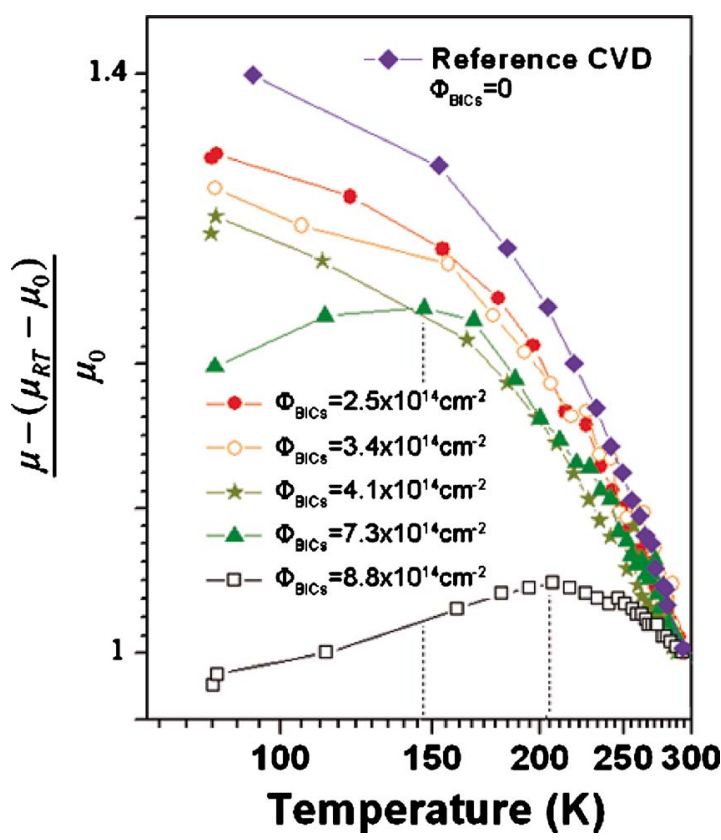

FIG. 4. (Color online) Normalized curves of drift mobility vs temperature for the five studied samples containing various concentrations of BICs and the CVD grown reference sample.

Table II) and the only parameter that changes between them is BICs concentration. As shown in Fig. 4, the temperature corresponding to the maximum mobility level is higher when BICs concentration increases. This clearly suggests that scattering centers due to BICs have a similar behavior to that of ionised impurities. In the case of very high BICs concentrations as for the $750{ }^{\circ} \mathrm{C}-15$ min annealed sample, scattering centers due to the BICs are so efficient that the expected mobility enhancement due to reduced lattice scattering is only weakly observed between RT and $200 \mathrm{~K}$ (cf. Fig. 4), leading to similar values of drift mobility at $\mathrm{LN}_{2}$ and $\mathrm{RT}$.

This temperature behavior of drift mobility and the linearity of the mobility coefficient $\alpha$ with BICs concentration reported in previous section (cf. Fig. 3) indicates that BICs can be considered as independent scattering centers, which limit drift mobility together with the other scattering mechanisms like lattice scattering and ionised impurities scattering. Thus, our results are consistent with Matthiessen's rule, to which an additional scattering center related to the BICs can be added

$$
\frac{1}{\mu_{d}}=\frac{1}{\mu_{\text {lattice }}}+\frac{1}{\mu_{\text {impurities }}}+\frac{1}{\mu_{\mathrm{BICs}}},
$$

where $\mu_{d}$ is the drift mobility, $\mu_{\text {lattice }}$ is the mobility associated to scattering from lattice vibration, $\mu_{\text {impurities }}$ is mobility contribution related to the scattering from ionised impurities, and $\mu_{\mathrm{BICs}}$ is the mobility contribution associated with the scattering from the BICs.

Finally, it is important to note that only by taking into account the impact of BICs on Hall effect measurements ${ }^{18}$ it was possible to show how they also directly affects hole drift mobility. 


\section{CONCLUSIONS}

In this work, we have investigated the impact of boron interstitial clusters BICs on the drift mobility of holes in high-dose boron-implanted ultrashallow junctions. We first presented a method to evaluate the BICs concentration in different samples which is based on the self-consistent interpretation of SIMS and Hall measurements. We defined a mobility coefficient, $\alpha$, reflecting the mobility degradation level in the presence of BICs. We evaluated this coefficient in several samples realized with the same implantation and preamorphisation conditions but submitted to different anneals leading to different BICs concentrations. $\alpha$ was found equal to unity in samples without BICs and decrease linearly when increasing BICs concentration: this indicates that these clusters are responsible of drift mobility degradation.

In order to determinate the mechanisms associated to the observed drift mobility degradation, temperature dependant Hall effect measurements (from $\mathrm{LN}_{2}$ to RT) were carried out. The observed behavior of drift mobility with temperature and the linearity of the mobility coefficient $\alpha$ variation with BICs concentration allowed us to consider BICs as independent scattering centers, which control drift mobility together with the other scattering mechanisms such as lattice scattering and ionised impurities scattering. BICs induced scattering can therefore be added to Matthiessen's rule as an additional scattering mechanism. Finally, these results show that, in addition to an impact on Hall effect measurements, BICs also have a direct impact on drift mobility.

\section{ACKNOWLEDGMENTS}

Part of this work has been carried out within the ATOMICS European Project (FP6, Contract No. 027152).

\footnotetext{
${ }^{1}$ International Technology Roadmap for Semiconductors (http:// www.itrs.net/, 2008 Edition).

${ }^{2}$ A. Agarwal, H. J. Gossmann, D. J. Eaglesham, L. Pelaz, S. B. Herner, D. C. Jacobson, and T. E. Haynes, Mater. Sci. Eng., A 253, 269 (1998).

${ }^{3}$ N. Cherkashin, M. Hytch, F. Cristiano, and A. Claverie, Solid State Phenom. 108-109, 303 (2005).

${ }^{4}$ F. Cristiano, X. Hebras, N. Cherkashin, A. Claverie, W. Lerch, and S. Paul, Appl. Phys. Lett. 83, 5407 (2003).

${ }^{5}$ W. Lerch, S. Paul, J. Niess, S. McCoy, T. Selinger, J. Gelpey, F. Cristiano, F. Severac, M. Gavelle, S. Boninelli, P. Pichler, and D. Bolze, Mater. Sci. Eng., B 124-125, 24 (2005).
}

${ }^{6}$ A. E. Michel, W. Rausch, and P. A. Ronsheim, Appl. Phys. Lett. 51, 487 (1987).

${ }^{7}$ S. Mirabella, E. Bruno, F. Priolo, D. D. Salvador, E. Napolitani, A. V. Drigo, and A. Carnera, Appl. Phys. Lett. 83, 680 (2003).

${ }^{8}$ S. Paul, W. Lerch, X. Hebras, N. Cherkashin, and F. Cristiano, Mater. Res. Soc. Symp. Proc. 810, 215 (2004)

${ }^{9}$ L. Pelaz, G. H. Gilmer, H. J. Gossmann, C. S. Rafferty, M. Jaraiz, and J. Barbolla, Appl. Phys. Lett. 74, 3657 (1999).

${ }^{10}$ F. Cristiano, N. Cherkashin, P. Calvo, Y. Lamrani, X. Hebras, A. Claverie, W. Lerch, and S. Paul, Mater. Sci. Eng., B 114-115, 174 (2004).

${ }^{11}$ A. D. Lilak, M. E. Law, L. Radic, K. S. Jones, and M. Clark, Appl. Phys. Lett. 81, 2244 (2002).

${ }^{12}$ A. Mokhberi, P. B. Griffin, J. D. Plummer, E. Paton, S. McCoy, and K. Elliott, IEEE Trans. Electron Devices 49, 1183 (2002).

${ }^{13}$ B. J. Pawlak, R. Surdeanu, B. Colombeau, A. J. Smith, N. E. B. Cowern, R. Lindsay, W. Vandervorst, B. Brijs, O. Richard, and F. Cristiano, Appl. Phys. Lett. 84, 2055 (2004).

${ }^{14}$ E. N. Shauly and S. Lachman-Shalem, J. Vac. Sci. Technol. B 22, 592 (2004).

${ }^{15}$ G. Masetti, M. Severi, and S. Solmi, IEEE Trans. Electron Devices 30, 764 (1983).

${ }^{16}$ W. R. Thurber, R. L. Mattis, Y. M. Liu, and J. J. Filliben, The Relationship Between Resistivity and Dopant Density for Phosphorus- and BoronDoped Silicon (U.S. Dept. of Commerce, National Bureau of Standards, Washington, D.C., 1981).

${ }^{17}$ T. Clarysse, J. Bogdanowicz, J. Goossens, A. Moussa, E. Rosseel, W. Vandervorst, D. H. Petersen, R. Lin, P. F. Nielsen, O. Hansen, G. Merklin, N. S. Bennett, and N. E. B. Cowern, Mater. Sci. Eng., B 154-155, 24 (2008).

${ }^{18}$ F. Severac, F. Cristiano, E. Bedel-Pereira, P. F. Fazzini, W. Lerch, S. Paul, X. Hebras, and F. Giannazzo, J. Appl. Phys. 105, 043711 (2009).

${ }^{19}$ Annual Book of ASTM Standard Electronics (II) F723-88 (American Society for Testing and Materials, Pennsylvania, 1991), p. 508.

${ }^{20}$ Y. Sasaki, K. Itoh, E. Inoue, S. Kishi, and T. Mitsuishi, Solid-State Electron. 31, 5 (1988).

${ }^{21}$ F. Severac, F. Cristiano, E. Bedel-Pereira, W. Lerch, S. Paul, and H. Kheyrandish, Mater. Sci. Eng., B 154-155, 225 (2008).

${ }^{22}$ P. Pichler, in Intrinsic Point Defects, Impurities, and Their Diffusion in Silicon, Computational Microelectronics, edited by S. Selberherr (Springer, Berlin, 2004)

${ }^{23}$ L. J. Van der Pauw, Philips Res. Rep. 13, 1 (1958).

${ }^{24}$ R. Baron, G. A. Shifrin, O. J. Marsh, and J. W. Mayer, J. Appl. Phys. 40, 3702 (1969).

${ }^{25}$ K. Seeger, Semiconductor Physics (Springer, New York, 2004), Vol. 9, p. 48.

${ }^{26}$ C. Canali, M. Costato, G. Ottaviani, and L. Reggiani, Phys. Rev. Lett. 31, 536 (1973).

${ }^{27}$ R. A. Logan and A. J. Peters, J. Appl. Phys. 31, 122 (1960).

${ }^{28}$ F. J. Morin and J. P. Maita, Phys. Rev. 96, 28 (1954).

${ }^{29}$ P. Norton, T. Braggins, and H. Levinstein, Phys. Rev. B 8, 5632 (1973).

${ }^{30}$ G. Ottaviani, L. Reggiani, C. Canali, F. Nava, and A. Alberigi-Quaranta, Phys. Rev. B 12, 3318 (1975) 